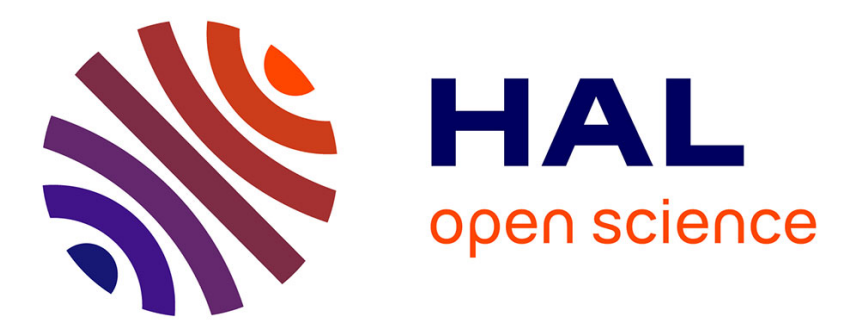

\title{
Familiarity Detection with the Component Process Model
}

\author{
Joseph P Garnier, Jean-Charles Marty, Karim Sehaba
}

\section{To cite this version:}

Joseph P Garnier, Jean-Charles Marty, Karim Sehaba. Familiarity Detection with the Component Process Model. 16th International Conference on Intelligent Virtual Agents (IVA 2016), Sep 2016, Los Angeles, CA, United States. pp.373-377, 10.1007/978-3-319-47665-0_36 . hal-01531558

\section{HAL Id: hal-01531558 https://hal.science/hal-01531558}

Submitted on 1 Jun 2017

HAL is a multi-disciplinary open access archive for the deposit and dissemination of scientific research documents, whether they are published or not. The documents may come from teaching and research institutions in France or abroad, or from public or private research centers.
L'archive ouverte pluridisciplinaire HAL, est destinée au dépôt et à la diffusion de documents scientifiques de niveau recherche, publiés ou non, émanant des établissements d'enseignement et de recherche français ou étrangers, des laboratoires publics ou privés. 


\title{
Familiarity Detection with the Component Process Model
}

\author{
Joseph P Garnier ${ }^{1}$, Jean-Charles Marty ${ }^{2}$, and Karim Sehaba ${ }^{3}$ \\ 1 Université de Lyon, CNRS Université Lyon 1, LIRIS, UMR5205, F-69622, France \\ joseph.garnierdiris.cnrs.fr \\ 2 Université de Lyon, CNRS Université de Savoie, LIRIS, UMR5205, F-69622, France \\ jean-charles.martyeliris.cnrs.fr \\ 3 Université de Lyon, CNRS Université Lyon 2, LIRIS, UMR5205, F-69676, France \\ karim.sehabadiris.cnrs.fr
}

\begin{abstract}
We propose a computational model for the Component Process Model (CPM) of Scherer, the most recent and the most complete model of emotion in psychology. This one proposes to appraise a stimulus through a sequence of sixteen appraisal variables dealing with a large number of its characteristics. As CPM is very abstract and high level, it is not really used in affective computing and no formal models exist for its appraisal variables. Based on the CPM, in this paper we propose a mathematical function for one appraisal variable detecting the familiarity of a perceived event according to the state of the cognitive component of an agent (goals, needs, semantic memory, and episodic memory).
\end{abstract}

\section{Introduction}

In our research work, we are particularly interested in motivation and immersion of users in numeric environments. Emotions play a critical role in processes such as rational decision-making, perception, human interaction, social relationships, human creativity and human intelligence. According to cognitive theories, an agent appraises a situation with respect to its knowledge, its goals, and other cognitive components. This appraisal takes place along multiple appraisal variables, such as familiarity (Is this event novel for me?), goal relevance (Is this situation good or not for me?), control (can I deal with this event?), and so on. This evaluation of an event leads to an emotion. One of these theories, called Component Process Model (CPM) [4], proposes to appraise an event with sixteen appraisal variables as opposed as most other theories as OCC or Lazarus that only have six to eight variables. A large number of appraisal variables can cover a large interpretation of an event according to a large number of points of view, and there is less risk to miss important factors of interpretation.

Among the existing models, no one proposes a domain-independent computational model with a formal description (as mathematical functions) of CPM's appraisal variables according to cognitive components as perceptions, goals, memory, motivation and knowledge of a cognitive agent. The most advanced work is probably PEACTIDM [2], proposing a computational model for CPM. However, it does not propose how to calculate appraisal variables but a way to compute mood and feeling, including its intensity, from emotion.

CPM proposes sixteen appraisal variables, the main purpose of this paper being to model the familiarity appraisal variable for relevance detection. Scherer proposes 
a process model describing how the appraisals are generate but he does not provide detailed explanations of all the data needed to the appraisals, and there is little or no guidance from the literature. We therefore decided to start with the "simplest" alternative. Our long-term strategy is to check by experimentation whether simple assumptions fall short. In such cases, we will thus improve complexity incrementally. Thus, this proposal is likely oversimplified, but it provides a starting point for future work.

\section{Appraisal process: Familiarity detection}

Familiarity with a perceived event event $\in E V T$ is directly related to the knowledge of the event's items, their associations, and the number of occurrences of these associations in the episodic memory .

In the episodic memory, events are encoded into memory traces as a vector where each element of an event is an item of this vector. Two traces are similar or different according to the number of their common characteristics. It is the association between spatio-temporal contextual items which makes a difference between an event (or a memory trace) and another one. In adulthood it is rare to be confronted with a new event's item or with an information completely new. Novelty (unfamiliarity) often lies in the association between various acontextuals known items. For example the novelty in the wife in the best friend's bed lies neither in the wife, nor the friend, nor the bed, but in the unfamiliar conjunction of the three [3]. So familiarity is determined by the number of new associations of a perceived event's items, compared to memory traces of episodic memory. Thus, to compute familiarity we check if an entity knows each item of a perceived event, then if it is the case, we count the number of occurrences of event's item associations. For instance, we formalize an event as a family set of three items: source $_{\text {evt }}$, action $_{\text {evt }}$, target $_{\text {evt }}$. Then we check in semantic memory if each item is known, if is is the case, we count the number of occurrences of event's items associations in episodic memory $\left\{\right.$ source $_{\text {evt }}$, action $\left._{\text {evt }}\right\}$, $\left\{\right.$ source $_{\text {evt }}$, target $\left._{\text {evt }}\right\},\left\{\right.$ action $_{\text {evt }}$, target $\left._{\text {evt }}\right\}$, and $\left\{\right.$ source $_{\text {evt }}$, action $_{\text {evt }}$, target $\left._{\text {evt }}\right\}$. Thus we define pair $_{\text {item }}=\{(x, y) \mid x \in$ evt $\wedge y \in$ evt $\wedge x \neq y\}$.

Function's characteristics. We used the following characteristics to create our familiarity function, according to what we explained before:

1. Limited range: familiarity value should be in the range $[0,1]$. 0 means an event is new and 1 means an event is completely familiar.

2. Beginning point: familiarity value should be 0 when an event does not exist in episodic memory or when an item of appraised event is unknown in semantic memory.

3. Event familiarity: familiarity value should be higher than 0 when the number of event occurrences is not 0 in episodic memory. Familiarity increases with experience.

4. Couples of event item familiarity: familiarity value should be higher than 0 when the number of occurrences of couples of event item is not 0 in episodic memory. Familiarity increases with experience.

5. Endpoint: a familiarity rate should be defined to control when an event is familiar.

6. Non-linear: according to [1] this function should be non-linear. 
Familiarity function. To construct our familiarity function, we begin with the characteristic 2 . If an item of the perceived event does not exist in semantic memory, familiarity value is 0 . For the other characteristics we propose an exponential function into two parts: the first one treats the event without considering its item pairs, and the second one treats each item pair of the event.

Before explaining our function, let us denote by $n b O c c(e v t, E P M)$ the number of occurrences of an event in episodic memory, and by $n b O c c((x, y), E P M)$ the number of times that action action $_{\text {evt }}$ has been experienced by source $_{e v t}$ or by target $_{\text {evt }}$, and the number of times that source $_{\text {evt }}$ interacted with target $_{\text {evt }}$ (reverse is true too).

Episodic memory stores a set of episodes that represent the life experience of the entity. Each episode contains a sequence of events appended during a context. A context is a fixed period of time and a location in the environment. The $n b O c c u()$ function counts the number of times an event or its pairs occurs in all episodes because familiarity is not context-sensitive. We define the familiarity function as:

$$
\begin{gathered}
\operatorname{appfun}_{\text {fami }}(\text { evt }, S M, E P M, G O A L)= \\
\left\{\begin{array}{l}
0, \text { if } \exists \text { item }:(\text { item } \in \text { evt } \wedge \text { item } \notin S M) \\
\left(1-\alpha^{\gamma . n b O c c(e v t, E P M)}\right)+\left(\beta . \alpha^{\gamma \cdot n b O c c(e v t, E P M)} \cdot\left(1-\alpha^{\gamma . a v g(e v t, E P M)}\right)\right), \text { else }
\end{array}\right. \\
\text { where } \operatorname{avg}(\text { evt }, E P M)=\frac{\sum_{\text {pair }_{i} \in \text { pair }_{\text {item }}} n b O c c\left(\text { pair }_{i}, E P M\right)}{\mid \text { pair }_{\text {item }} \mid}
\end{gathered}
$$

According to characteristic 6 , we propose an exponential function formed with two exponential functions ${ }^{4}$ where familiarity is between 0 and 1 value (characteristic 1). Exponential functions with a negative sign are interesting because they passes through 0 and have an asymptotic value equals to 1 . The first exponential function (characteristic 3) treats the event familiarity in a whole (in other words treats associations between all event's items), where $\alpha$ is a positive real constant such that $\alpha<1$. $\alpha$ can be seen as a "familiarity rate" determining how the familiarity appraisal value increases with experience (characteristic 5). In figure 1 this function is in red. The second exponential function (characteristic 4) treats the familiarity of each pair of event's items. $\beta$ is a real positive constant such that $\beta<1$, seen as the contribution of the second function to the global familiarity function. To have a global familiarity value not higher than 1 (characteristic 1), we multiply $\beta$ by the complement of first exponential function (which treats the event familiarity of associations between all event's items). Familiarity of pairs associations is computed in the same way as the event familiarity. We use the function avg (evt, EPM) to "merge" familiarity of each pair. An average function has been chosen for several reasons:

- We can't use a multiplication because if a pair is not in episodic memory while the others are, familiarity should not be 0 ;

- We can't use just a sum of occurrences because familiarity of pairs (second power function) will increase too quickly with regard to the first power function, we would have weighted the first one but this value would be hard to define in a general context.

\footnotetext{
${ }^{4}$ please note that $a^{x}=\exp _{a}(x)=e^{x \cdot \ln (a)}$.
} 
Finally, we weight $n b O c c()$ and $\operatorname{avg}()$ values with $\gamma$, a real positive constant such that $\gamma \leq 1$, to control the rate of growth of the familiarity (in our experience, agents become too familiar too quickly otherwise, the value chosen is purely empirical), but this value is optional and can be equals to 1 . In figure 1 the second exponential function is in blue, the first one is in red, and the general familiarity function is in green.

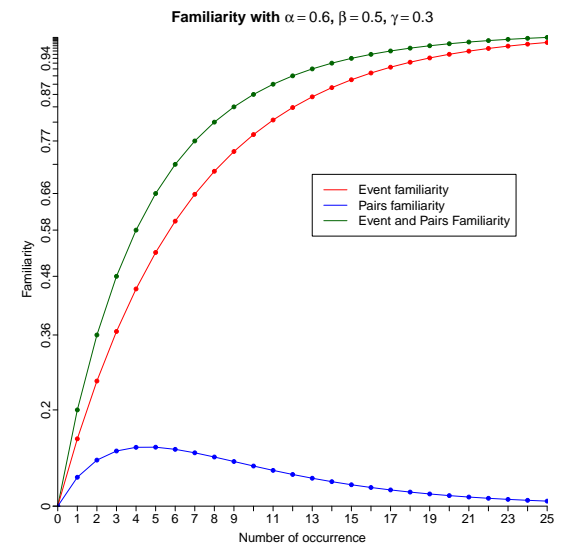

Fig. 1. Familiarity function. The red function is the familiarity of the event without considering its item pairs, and the blue function is the familiarity of each item pairs of the event. The combination of the red function and the blue one, gives the green function which is the familiarity of a perceived event, used by the appraisal process. Here $x=n b O c c()=\operatorname{avg}()$ but in a real situation, the number of occurrences of an event will often be different of average of the number of occurrences of pairs.

Future Work In this paper we have presented a computational mathematical functions for familiarity detection. We will describe others appraisal variables for relevance in another paper.

Acknowledgements The authors would like to thank the studio of video games named Artefacts Studio and the ANRT, and Marie-Neige Chapel for her contributions.

\section{References}

1. Logan, G.D.: Toward an instance theory of automatization. Psychological Review 95(4), 492 527 (1988)

2. Marinier, R.P., Laird, J.E., Lewis, R.L.: A computational unification of cognitive behavior and emotion. Cognitive Systems Research 10(1), 48-69 (2009), http://linkinghub.elsevier.com/retrieve/pii/S1389041708000302

3. O'Keefe, J., Nadel, L.: The Hippocampus as a Cognitive Map. Oxford University Press (1978)

4. Scherer, K.R.: Appraisal Considered as a Process of Multilevel Sequential Checking. In: Appraisal processes in emotion: Theory, methods, research, pp. 92-120. Oxford University Press (2001) 